\section{RETOS Y TENDENCIAS DEL OCIO DIGITAL: TRANSFORMACIÓN DE DIMENSIONES, EXPERIENCIAS Y MODELOS EMPRESARIALES*}

\author{
Ercilia García Álvarez \\ Universitat Rovira i Virgili \\ Facultad de Geografía y Turismo. \\ Departamento de Gestión de Empresas. Av. de la Universitat, 1. \\ 43204 Reus \\ mariaercilia.garcia@urv.cat \\ Jordi López Sintas \\ Universitat Autònoma de Barcelona, Facultat d'Economia i Empresa, \\ Departamento de Economía de la Empresa. Edifici B, \\ 08193-Bellaterra (Cerdanvola del Vallès) \\ jordi.lopez@uab.es

\begin{abstract}
Alexandra Samper Martínez
Universitat Rovira i Virgili. Facultad de Geografía y Turismo. Departamento de Gestión de Empresas. Av. de la Universitat, 1. 43204 Reus

alexandra.samper@urv.cat
\end{abstract}

\begin{abstract}
Digital technologies are transforming leisure and the way it is organized. Despite its importance, few studies have addressed the study from the academic world. In this paper, we make an extensive literature review on leisure and digital technologies, in order to contribute to knowledge by developing an interpretive framework of digital entertainment. We suggest that digital technologies bring a new dimension to leisure, altering its traditional dichotomous properties, placing individuals and businesses between a holistic or separate view of the natural and digitally built worlds. In the first case blurs the boundary between the dichotomous properties of leisure and work, not in the second. Both conceptions are evident in different degrees in which changes shape within individuals and on how businesses solve the challenges appeared.
\end{abstract}

KEY WORDS: Digital entertainment; generation divide; social divide; social interaction; mobile identity; gender; realizing digital entertainment; digital traditional entertainment; leisure co-production.

\section{INTRODUCCIÓN}

Las tecnologías digitales no sólo han facilitado el desarrollo de nuevas actividades de ocio (como los juegos digitales que conviven con los tradicionales, los espacios
CHALLENGES AND TRENDS

IN DIGITAL LEISURE:

TRANSFORMATION OF

DIMENSIONS, EXPERIENCES

AND THE NEW BUSINESS

MODELS

RESUMEN: Las tecnologias digitales están transformando el ocio y la manera en la que se organiza. A pesar de su importancia, son escasos los trabajos que han abordado su estudio desde el mundo académico. En este artículo realizamos una revisión extensa de la literatura sobre ocio y tecnologías digitales con el objeto de contribuir al conocimiento elaborando un marco interpretativo del ocio digital. Proponemos que las tecnologías digitales aportan una nueva dimensión al ocio que altera sus propiedades dicotómicas tradicionales, situando a individuos y empresas entre una comprensión holística o separada de los mundos naturales y los construidos digitalmente. En el primer caso se borran los limites entre las propiedades dicotómicas del ocio y el trabajo, no asi en el segundo. Una u otra concepción se evidencia en los diferentes grados en los que las transformaciones tienen lugar entre los individuos y en cómo las empresas resuelven los retos que aparecen.

PALABRAS CLAVE: Ocio digital; brecha generacional; brecha social; interacción social; identidad móvil; género; materialización ocio digital; digitalización ocio tradicional; coproducción ocio.

de interacción social en la red, reproductores de música y audiovisuales, lectores de libros electrónicos, cámaras fotográficas digitales, etc.), sino también la transformación de las actividades tradicionales y su organización. A pesar de la importancia de las transformaciones y de su creciente 
capacidad de crear riqueza (en España, el volumen de negocio de los juegos digitales supera al del audiovisual), son escasas las investigaciones que han abordado el estudio del ocio digital desde una perspectiva académica (Bryce, 2001; Mokhtarian, Salomon y Handy, 2006; Juniu, 2009).

En este trabajo estudiamos las transformaciones que las tecnologías digitales están produciendo en la experiencia de ocio de los individuos y discutimos los retos a los que se enfrentan las empresas si quieren continuar creando valor para la sociedad. Para ello, primero analizamos cómo la naturaleza de las tecnologías digitales altera las dimensiones o propiedades tradicionales del ocio. Esta manera de proceder nos permite determinar las diferencias que se observan en la práctica del ocio, y el ocio digital en particular, debido a que los individuos difieren respecto a cómo interpretan su relación con los mundos construidos digitalmente y con los naturales, ya sean considerados como dos ámbitos separados o como uno solo, tal y como afirma Kozinets $(2010,1)$ cuando sugiere que "nuestros mundos sociales se están tornando digitales".

Seguidamente, presentamos cómo la diferente exposición a las tecnologías digitales, y su uso, incrementa la brecha generacional y la social. A continuación, abordamos la influencia del ocio digital en la interacción social, en la construcción de una identidad, y en la producción y reproducción de la construcción social de género. Finalmente, discutimos las implicaciones de la materialización del ocio digital y la coproducción de la experiencia de ocio entre individuos y empresas como principales retos empresariales dentro del sector, derivados de las tendencias actuales del ocio digital.

\section{Marco interpretativo}

Las tecnologías digitales están transformando el ocio, afectando tanto al tradicional como a las nuevas manifestaciones digitales (Bryce, 2001; Mokhtarian, Salomon y Handy, 2006), aunque la intensidad de los cambios varía según los grupos sociales. Antes de trazar las transformaciones de la experiencia de ocio y los retos que estas tendencias suponen para las empresas, exponemos cómo las tecnologías digitales alteran las propiedades tradicionales del ocio.
Moktarian, Salomon y Handy (2006) argumentan que todas las definiciones del ocio propuestas por diversos autores son restrictivas debido a que privilegian algunas propiedades del ocio frente a otras (Kelly, 1978; Gershuny y Fisher, 1999; Wilson, 1980). No obstante, cuando analizamos las diferentes aproximaciones se suelen destacar ciertas propiedades del ocio: 1) la dimensión espacial; 2) la dimensión temporal; 3) la dimensión sustantiva: qué actividades son catalogadas como ocio; 4) la dimensión cultural: qué significa el ocio para las personas; 5) la dimensión social: cómo se relacionan las personas a través del ocio; y 6) la dimensión económica: el ocio como negocio. En el contexto actual las tecnologías digitales no sólo han facilitado el desarrollo de nuevas actividades de ocio sino que han transformado las actividades tradicionales, y alterado las dimensiones del ocio incorporando una nueva con dos valores opuestos: hoy la experiencia de ocio puede tener lugar en un mundo natural o en otro construido digitalmente. Esta nueva dimensión facilita la interpenetración de las dimensiones tradicionales.

Por ejemplo, en el año 2008 la encuesta realizada por el Pew Research Center para conocer el consumo de noticias reveló la existencia de una asociación entre el acceso a Internet en el trabajo y el consumo de noticias digitales, lo cual no hacía más que reforzar los resultados encontrados un año antes en Europa y Argentina (Boczkowski, 2010). Esta convergencia pone de manifiesto que los patrones de lectura de las noticias están cambiando debido a la penetración del tiempo de ocio en el espacio de trabajo como consecuencia de la nueva dimensión del ocio: la digitalización de su experiencia.

Antes de la penetración del tiempo de ocio en el espacio de trabajo (gracias a los mundos construidos digitalmente), las investigaciones revelaban la existencia de unas prácticas estructuradas secuencial y dinámicamente: escuchar la radio y leer diarios era una actividad matinal que tenía lugar en el hogar y la TV vespertina (Bogart, 1989). Esto es, la lectura de noticias estaba integrada en las rutinas de la vida cotidiana doméstica, tenía lugar fuera del trabajo. Ahora con la penetración del tiempo de ocio en el espacio de trabajo emerge un patrón discontinuo en la lectura de noticias: se realizan varias visitas diarias a páginas web de noticias, las primeras son metódicas, las siguientes no (Boczkowski, 2010). 
A pesar de la posible interpenetración del espacio y el tiempo, no siempre ocurre, ya que depende de cómo los individuos y empresas interpreten ambos mundos, el construido digitalmente y el natural: (1) como espacios separados y opuestos, o bien (2) como la ocasión de interpenetrar las dimensiones del ocio que antes estaban separadas. Si adoptamos la primera visión aparecen conceptos como ocio online, ocio virtual, etc., que expresan una visión dicotómica: el primero privilegia la conexión digital (estar conectado o no estarlo) y el segundo privilegia el mundo social natural (lo virtual no es real, lo físico, sí). Como veremos al abordar los retos empresariales, estas concepciones opuestas también se presentan en los modelos de negocio del ocio.

Interpretar el ocio y el ocio digital como opuestos y aislados se desprende de la oposición entre los dos mundos, el natural y el construido digitalmente, pero olvida que ambos están conectados, es el mismo individuo quien interactúa en ambos mundos, y con frecuencia, con las mismas personas con las que se encuentra cara a cara. En cambio, concebir el ocio de manera holística privilegia el estudio del individuo y su experiencia contextual, independientemente de que disfrute de sus actividades de ocio durante su tiempo libre en el mundo natural $o$ en el digital. Adoptar una u otra visión del papel de las tecnologías digitales en la experiencia de ocio tiene implicaciones, tanto en la investigación del ocio digital como en la vida ordinaria de las personas y en los modelos de negocio.

Por ejemplo, Griffiths y Light (2008) estudian el comportamiento ético de los jugadores de Second Life y encuentran que un grupo de individuos suele engañar a los otros jugadores. Los primeros argumentan que el juego no es real y que por lo tanto no importa y pueden comportarse de maneras diferentes a como lo harían en el mundo social en el que interactúan cara a cara. Esta es una interpretación que enfatiza la separación de los mundos, virtual (digital) frente a real (natural). Pero desde una concepción holística de la experiencia de ocio, el comportamiento de los defraudadores se interpretaría de otra manera. Los individuos son los mismos en un mundo u otro, el natural y el construido digitalmente, pero cambian las propiedades estructurales de ambos y con ello el impacto negativo de su comportamiento.
Esta concepción holística, donde las tecnologías digitales añaden una nueva propiedad a la experiencia de ocio, nos permite identificar más fácilmente las transformaciones que se están produciendo e identificar las brechas que aparecen en la manera en la que el ocio tiene lugar. Estas brechas tienen su origen en los diferentes grados de interpenetración entre los mundos sociales naturales (interacción personal) y los construidos digitalmente (interacción personificada), así como a la posición del individuo en la sociedad. De esta manera, interpretamos el ocio digital en Internet no como un estar conectado (ocio online) frente a no conectado (ocio offline), sino como seguir conectado en el mundo social digitalmente construido; no como un ocio virtual frente a un ocio real, sino como un nuevo espacio donde continuar las relaciones sociales.

\section{Transformaciones dE LA EXPERIENCIA DE OCIO}

\section{La brecha generacional en el ocio digital}

Aunque los adultos dispongan del capital económico para utilizar las tecnologías digitales con profusión, son los jóvenes los principales usuarios durante su tiempo de ocio (Hargittai y Hinnant, 2008). Esas diferencias en el uso de las tecnologías digitales como ocio evidencian diferentes grados de penetración entre ambos mundos, el natural y el digitalmente construido, que toma su máxima expresión cuando ambos se solapan y logran que los espacios de trabajo y ocio y los tiempos de trabajo y ocio se fusionen, uniendo lo que la sociedad industrial separó, como ocurre en el caso de los hackers (Turgeman-Goldschmidt, 2005).

Dos conceptos propuestos por Prensky (2001a y b) maximizan la diferencia de la brecha generacional al hablar de los nativos digitales (los jóvenes actuales que han nacido "enchufados" al mundo digital) y los inmigrantes digitales (nacidos en el mundo natural pero que han tenido que emigrar al digital), dos grupos entre los cuales podemos encontrar innumerables combinaciones intermedias.

La evidencia muestra que los nativos digitales habituados a los juegos digitales dedican mucho tiempo libre a desarrollar las habilidades necesarias para poder "subir de nivel" en los juegos, hábito que más tarde les facilita de manera "natural" desarrollar prácticas de ocio similares como, por

ARBOR Vol. 188754 marzo-abril [2012] 395-407 ISSN: 0210-1963

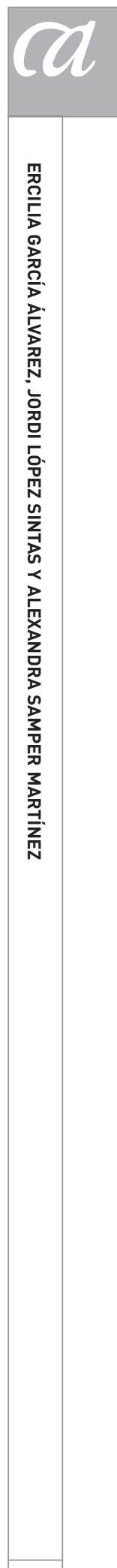

397 
ejemplo, la fotografía digital, que a su vez incrementa su interacción social presencial y personificada (Cox, Clough y Marlow, 2008). La constancia en el desarrollo de habilidades y competencias, de hecho, transforma una forma de vivir el juego, de ocio ocasional a ocio serio (Stebbins 1992; 1997), donde las recompensas se obtienen con "seguridad" si se realiza el esfuerzo necesario (De Kort, IJsselsteijn y Gajadhar, 2007; Johnson, 2009; Prensky, 2001a y b).

Esta forma de proceder de los nativos digitales parece ser la causa de que se impacienten con las prácticas habituales en el mundo natural. Se han habituado a la resolución de problemas y a desarrollar habilidades según sean necesarias para resolver o para ampliar sus temas de interés, así se habitúan a la multitarea (mientras se localiza el conocimiento necesario para resolver una tarea se va realizando otra, y cuando lo obtienen retoman la tarea anterior de forma iterativa). Este proceder choca con el pensamiento lineal del mundo natural que compartimenta el conocimiento y lo aisla de su contexto (Jones et al., 2010).

En cambio, los inmigrantes digitales, para los que las nuevas tecnologías digitales interactivas son su segundo hogar, se comportan de manera diferente. Habituados al pensamiento lineal, a un mundo con compartimentos aislados (espacio de trabajo frente a espacios de ocio, tiempo de trabajo, tiempo de ocio, actividad de trabajo, actividad de ocio), los mundos construidos digitalmente no son más que otra complejidad añadida donde sus habilidades no son las más adecuadas para moverse "como pez en el agua", como un nativo digital. Es más, como veremos, las tecnologías digitales y en especial las que tienen lugar en Internet, potencian especialmente la interacción social, una actividad que no es tan importante para los adultos que ya han formado unas relaciones familiares (López, Filimon y García, 2011).

Las investigaciones muestran que los nativos digitales buscan en la Red información de manera activa, tienen una gran exposición a nuevos canales de comunicación simultáneamente y no temen al riesgo ni al cambio (su mundo digital está en constante transformación), y disfrutan de las nuevas tecnologías durante su tiempo de ocio (Buse, 2009). Respecto a las actividades de ocio, Johnson y Kaye (2003) argumentan que los nativos digitales son más propensos a usar Internet como entretenimiento (chat, mensajes instantáneos, realizar búsquedas por diversión, bajarse música, o visitar lugares en Internet relacionados con la música, libros y otras actividades de ocio). Por ello, algunos autores proponen que para los nativos digitales la Red es un espacio "natural", aunque está construido tecnológica y socialmente, donde tienen lugar las actividades de ocio y la interacción social (Livingstone, 2002). En cambio, los inmigrantes digitales difieren en los significados que le dan al binomio ocio y tecnologías digitales y en las actividades que realizan, lo que ha dado lugar a una brecha entre generaciones (Selwyn, 2004).

\section{Brecha social en el ocio digital}

Si bien la brecha generacional sugiere que las transformaciones que observamos en el ocio difieren según generaciones, la brecha social tiene su origen en las diferencias de capital (económico, cultural y social) que se observan dentro de las generaciones. La brecha social pone de manifiesto que la libertad de elección y acceso al ocio está limitada socialmente, como proponen DiMaggio y Hargittai (2001), lo cual contradice ciertos aspectos de la noción de ocio propuestos por Iso-Ahola (1997) y Neulinger (1974). Por ejemplo, el nivel de ingresos es una condición necesaria pero no suficiente para estar en posición de permitirse y mantener el coste de la tecnología (DiMaggio y Hargittai, 2001), para poder ser libre de elegir participar en las actividades de ocio digitales.

La brecha social en el disfrute del ocio digital se manifiesta en dos fases. Primero, en el acceso a la tecnología (Bucy, 2000; DiMaggio et al., 2001), y después en su uso (DiMaggio et al., 2004; Zillien and Jäckel, 2007), aunque es difícil separar una de otra: como dicen los jóvenes expertos entrevistados por Johnson (2009), "para estar a la última hace falta dinero" (p. 194). Lo cual significa que no sólo es el acceso, sino el acceso a qué tecnologías digitales y después a qué ocio éstas te dan acceso. Así, a medida que el índice de penetración de las nuevas tecnologías se incrementa (Hargittai y Hinnant, 2008), una vez se ha garantizado el acceso, debemos fijarnos en los diferentes usos de las nuevas tecnologías (Mäntymäki y Merikivi, 2010). Es decir, en función de los usos, nos encontramos con diferencias sociales en la forma de percibir el ocio.

\section{Interacción social durante el tiempo de ocio}

Las primeras interpretaciones de las implicaciones sociales del uso de los juegos digitales e Internet en el ocio fueron 
negativas, pronosticando una reducción en la interacción social (Kraut et al., 1998) y en el capital social (Putnam, 2000). La evidencia reciente, no obstante, contradice tales razonamientos; sugiere que los juegos digitales y la interacción en Internet dan lugar a una mayor interacción social personal (De Kort, IJsselsteijn y Gajadhar, 2007; Kraut et al., 2002), e incluso personificada (Mennecke et al., 2011).

Así, los juegos digitales en solitario, contrariamente a lo que uno esperaría, producen interacción social. Carr et al. (2004) encuentran evidencias de juego cooperativo entre amigos activamente implicados en el juego. La interacción social tiene lugar entre el que juega y los que están mirándolo, compartiendo la experiencia de jugar, amplificando los aspectos emocionales de la experiencia de formar parte de un grupo de jugadores. Este efecto tiene lugar, según la evidencia, tanto en contextos públicos de juego como privados y extiende los resultados previos encontrados al investigar sobre ver la TV en familia (De Kort, IJsselsteijn y Gajadhar, 2007).

Se sugiere que los niños que juegan regularmente a los videojuegos tienen el mismo número de amigos que los que no juegan con tanta frecuencia, incluso se propone que estos jugadores incrementan los contactos sociales y que suelen encontrarse con mayor asiduidad con otros compañeros al salir de la escuela (Colwell, Grady y Rhaiti, 1995). Los juegos aportan no sólo beneficios cognitivos a los niños sino también afectivos y sociales (Calvert, 2005 y Gunter, 2005).

Por otro lado, las tecnologías digitales han transformado la noción de familia, ahora no es el grupo familiar la pieza importante para estudiar la interacción social sino el individuo. La proliferación en los hogares de aparatos de TV, de juegos digitales que rivalizan con la TV, de ordenadores personales que compiten con las consolas de juegos, de ordenadores versus consolas conectadas a la red, etc., ha incrementado las diferencias internas entre los individuos de una familia, transformando la "foto" familiar en un puzzle formado por piezas individuales de diferentes "colores". Ahora las familias se parecen más a redes, más o menos densas, que no a grupos sociales homogéneos. Esas mismas tecnologías que los separan, ahora se utilizan para recomponer la familia: las tecnologías digitales sociales (teléfono móvil, mensajería en grupo, correo electrónico, redes sociales) se adoptan para compartir experiencias, sincronizar agendas, organizarse, comunicarse, coordinar su tiempo de ocio e interacción social (Kennedy y Wellman, 2007; Jupp y Bentlley, 2001). Las diferencias, no obstante, existen también entre familias en la medida en la que éstas difieren en su forma de ver los mundos naturales y los construidos digitalmente, como mundos separados o interpenetrados. Para las primeras, las tecnologías digitales separarán a los individuos; para las segundas, los unirán, como ha mostrado la investigación de EnglishLueck (1998) al estudiar a las familias de Silicon Valley, California (EUA).

\section{Ocio digital e identidad}

Usar el teléfono móvil, la mensajería instantánea, las redes sociales, escuchar y compartir música digital a través de Internet, son actividades que especialmente los jóvenes desarrollan durante su tiempo de ocio, no sólo para entretenerse sino también para construir su identidad social (Schroeder, 2010). Propone Stald (2011) que el teléfono móvil tiene dos cualidades interdependientes para los jóvenes: (1) una función comunicativa (contenido), y (2) otra social (mantener el contacto y construir una identidad). De ambas, probablemente la segunda, es la que aporta más valor para la construcción de esa identidad móvil, concepto que tiene un doble sentido, el de la movilidad de la propia tecnología digital utilizada y el sentido de provisionalidad que la identidad tiene para los adolescentes (Abbott-Chapman y Robertson, 2002).

Los mensajes electrónicos parecen estar vinculados a la programación del ocio social, especialmente entre los más jóvenes, según los resultados obtenidos por Campell y Kwak (2010) y Leung (2001); las Ilamadas telefónicas, en cambio, se asocian al tiempo libre dedicado a la interacción personal; el intercambio de música está relacionado con el dispositivo Bluetooth del terminal telefónico, una de las principales actividades de ocio de los jóvenes en general y de los iranies debido a que su gobierno controla el contenido televisivo e Internet (Prensky, 2001b y Nikman, 2010). Todo ello fortalece la construcción de una identidad social a través de compartir símbolos y significados: decimos lo que somos con lo que hacemos durante el tiempo de ocio (Douglas y Isherwood, 1996). Las redes sociales hacen pública una identidad que se va construyendo con los materiales de la experiencia vivida 
durante nuestro tiempo de ocio: espacios digitales como Facebook (la transformación digital del anuario del colegio puesto a disposición de mucha gente), Flickr (compartir la pasión por la fotografía digital), Hoteles Habbo (Mäntymäki y Merikivi, 2010), Twitter (la mensajeria en grupo), AmericaOnLine.com (Jupp y Bentley, 2001), etc., no hacen más que romper con los límites espaciales (geográficos) y temporales de actividades sociales tradicionales y sirven para seguir conectado, tener una presencia social digital en espacios públicos y privados, de trabajo y de ocio o en otros hogares, mantener un diario personal público (Leung, 2009). Además de facilitar la construcción de una identidad social, la red social permite durante el tiempo de ocio resaltar algunas aficiones o rasgos de los individuos que quedan ocultas en los mundos naturales donde mantienen una presencia social (Orchard y Fullwood, 2010).

Tan ávidos están los jóvenes de construirse un lugar en el mundo que Patterson (2011) sostiene que los adolescentes usan Facebook de manera socialmente adictiva, aunque también selectiva. Los adolescentes expertos en las nuevas tecnologías de la información y la comunicación, investigados por Johnson (2009), muestran un elevado grado de interacción selectiva: la construcción de una identidad social requiere relacionarse con otros privilegiados pero también con otros menos afortunados, yendo la interacción asociada a diferentes actividades lúdicas. Después, las afinidades temáticas como la fotografía (Cox, Clough y Marlow, 2008), genealogía (Fulton, 2009), etc., reemplazan a las geográficas (Holderness, 1994).

\section{Ocio digital y género}

Los estudios sugieren que hombres y mujeres difieren en el uso de las tecnologías utilizadas en el ocio y en sus concepciones de los mundos naturales y los construidos digitalmente (Cassell y Jenkins, 1998, y Martinson, Schwartz y Vaughan, 2002). Estas diferencias se basan, según Kennedy, Wellman y Klement (2003), en sus definiciones de género y en la estructura de las tareas del hogar. Por un lado, sugieren, las mujeres dedican menos tiempo a los juegos digitales porque tienen mayores responsabilidades domésticas que los hombres, por otro, son distintas las expectativas acerca de lo que se espera que hagan en su tiempo libre.

Según el marco interpretativo que hemos presentado, cabría esperar que la interpenetración de los espacios socia- les presenciales con los digitales personificados permitiera que no sólo se incrementara el tiempo de ocio de las mujeres sino también la interacción social y el ocio tradicional, e incluso que los espacios sociales digitales proporcionen el escenario necesario donde desafiar las definiciones tradicionales de lo que se espera que la mujer haga durante su tiempo de ocio (Delamere y Shaw, 2008).

Por otro lado, dado que las mujeres tradicionalmente se han encargado de ser las comunicadoras y forjadoras de las redes sociales de la familia (Robinson y Godbey, 1997), uno esperaría que utilizasen las tecnologías digitales durante su tiempo de ocio para comunicarse con más intensidad (Martinson, Schwartz y Vaughan, 2002). Y así es, según la evidencia proporcionada por la investigación de Kennedy y Wellman (2007), las mujeres creen que sus familias están más unidas gracias a Internet, usándolo además para incrementar sus contactos personales, reforzar sus relaciones y programar su ocio; en cambio, los hombres prefieren utilizarlo para encontrar a desconocidos que compartan sus intereses. Incluso parece que hombres y mujeres difieren en su interpretación de las tecnologías digitales, para los hombres son un juego, para las mujeres una herramienta para mantener su interacción social, aunque las diferencias parecen reducirse con el tiempo (Kennedy y Wellman, 2007; y Buse, 2009).

En el caso de los juegos digitales podemos ver que las concepciones tradicionales de género, los roles asignados a hombres y mujeres, se reproducen en el contenido de los juegos. Los juegos digitales parecen ser dominio del género masculino, no sólo por el contenido de los mismos programas sino también por el comportamiento que se espera de un jugador (Kiesler et al., 1985, y Pereira, 1994). Los adolescentes, además, suelen empezar a jugar a edades más tempranas y lo hacen de un manera más regular, favoreciendo los juegos de deportes y los violentos (Griffiths, 1997). No obstante, el espacio social digital también libera a las mujeres de la presión social que las conduce a reproducir las concepciones tradicionales y les permite desafiarlas (Jansz y Martens, 2005). Evidentemente, la capacidad para tal desafío parece depender del capital (económico, cultural y social) de las mujeres, como han puesto de relieve Delamere y Shaw (2008). Esto es, las relaciones de género dan forma a la tecnología y la tecnología da forma a las relaciones de género (Henwood, 1993). 


\section{RetOS EMPRESARIALES A LAS TRANSFORMACIONES DEL OCIO}

\section{La digitalización del ocio tradicional}

Los mundos construidos digitalmente también han alterado los modelos tradicionales del ocio como negocio, pero las empresas han reaccionado separando los modelos de negocio, por un lado las expresiones materiales de las actividades de ocio y por otro las digitales. La industria del ocio se enfrenta al desafío que supone transformar experiencias de ocio tradicionales en digitales y encontrar un nuevo modelo de negocio que encaje en la manera en la que los individuos interpretan las producciones tradicionales y las digitales (Ritzer y Jurgenson, 2010).

Los libros electrónicos empiezan a inundar los hogares, pero se concibe como un producto distinto con muchas limitaciones para el usuario: uno compra el libro material o bien el digital, y si se quieren ambos soportes hay que pagar dos veces (incluso buena parte de libros electrónicos se comercializan con numerosas restricciones en su uso). Esta concepción segmenta los mercados según el criterio de materialización de las expresiones culturales y favorece el desarrollo de la brecha generacional. Du y Martin (2008) sugieren que las tecnologías digitales están cambiando la manera en la que la gente lee; Roberts, Foehr y Rideout (2005), proponen que entre los jóvenes existen diferencias en la exposición a los medios y en especial en el consumo de diferentes formatos digitales.

En el caso de los audiolibros sucede algo parecido. Uno compra la versión analógica en una librería o bien la descarga de Internet, pero los criterios de categorización del producto no son los mismos en ambas plataformas lo cual confunde al consumidor; se hace necesario normalizar los criterios de catalogación de los libros materiales y de Ios digitales: etiquetas que adviertan del tiempo total, la calidad de la grabación, voz sintética o no, etc. (Engelen, 2008). Se ha empezado a señalar que es necesario un cambio en la concepción de los mercados, concebirlos de manera holística; que si compras un libro material dispongas también de sus expresiones digitales (Cruz, 2011; Engelen, 2008).

En la industria discográfica, por ejemplo, se ha demostrado que la batalla por mantener el modelo tradicional de negocio y limitar la distribución digital de la música está perdida. Así lo han entendido intérpretes como el grupo americano Metallica, que después de litigar contra Napster decidió afrontar el reto digital de manera proactiva; ahora no sólo distribuye su música digitalmente en la Red sino que también ha entrado con su música en el mundo de los videojuegos. La evidencia proporcionada por Khouja y Wang (2010) sugiere que las redes para compartir música en Internet afectan principalmente a la distribución tradicional, pero que la existencia de un canal de distribución digital en Internet reduce el grado en el que los individuos comparten esa misma música a través de las redes de intercambio social.

Los grandes estudios cinematográficos también han reaccionado oponiendo resistencia. Por ello no es de extrañar que las iniciativas de distribución digital hayan llegado de la mano de empresas ajenas a los estudios: iTunes, Amazon o Google, son ejemplos. Algunas iniciativas nacionales como Filmotech.com ya ofrecen el acceso en streaming "a la carta" como alternativa legal y de calidad (FICOD, 2008). Sin embargo, nos topamos de nuevo con una falta de adaptación al mercado por parte de los proveedores y políticas públicas de regulación que imposibilitan el lanzamiento de nuevos formatos, especialmente en España.

La música y el cine están experimentando otro cambio a raíz de la popularización de un nuevo formato que nos propone disfrutar de sus expresiones digitales sin tener que descargarlas (streaming), iniciativas como Spotify, Google, iTunes, y Amazon han liderado la innovación. Paralelamente los creadores e intérpretes también han modificado su forma de producir y distribuir sus producciones musicales, gestionar sus derechos de autor, sin necesidad de estar afiliado a una compañía discográfica. Todo ello no son más que evidencias de las diferentes respuestas de creadores, productoras y distribuidoras, a los desafíos propuestos por las tecnologías digitales. Respecto a los consumidores, las nuevas tecnologías les han ofrecido más capacidad de elección de sus actividades de ocio, al mismo tiempo que se ha reducido el valor de la intermediación tradicional.

Incluso la interacción social durante el tiempo de ocio que tiene lugar en los mundos naturales también se ha transformado en digital, y han dado lugar a mundos virtuales como Second Life que, según su fundador, Philip Rosedale (2007), funciona en más de 3.000 servidores y todos los meses

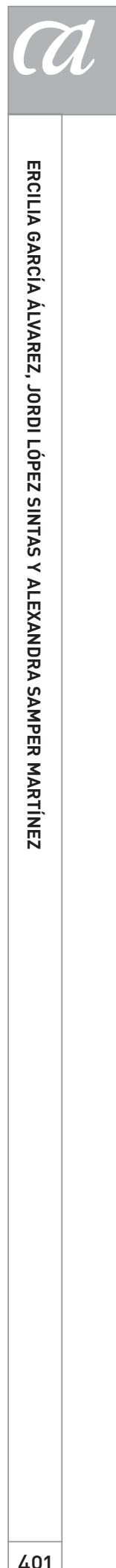

doi: 10.3989/arbor.2012.754n2011
ARBOR Vol. 188754 marzo-abril [2012] 395-407 ISSN: 0210-1963 
sus usuarios gastan cerca de 5 millones de dólares en este mundo virtual social. Es más, Second Life ha sido capaz de crear un sistema de fertilización cruzada entre el espacio digital y el físico (Bretonès et al., 2010); por ejemplo, yo como secondlaifiano/a puedo disponer de una tienda de zapatos en el mundo social construido digitalmente y vender productos digitales por los que otros pagarán realmente un precio material, pero además como también formo parte de un mundo social natural donde interactúo personalmente, puedo disponer simultáneamente de una tienda de zapatos donde distribuir los mismos modelos que ofrezco en mi tienda en Second Life. Toda una visión holística de los mundos natural y digital, claramente diferente a los modelos de negocio de los libros electrónicos, música y audiovisuales, aunque los consumidores sigan anclados en el mundo natural, como han sugerido Siddiqui y Turley (2006).

\section{La materialización de las actividades de ocio digital}

Con el objeto de incrementar la rentabilidad, las actividades de ocio de origen digital han seguido el camino opuesto. Los estudios Disney fueron los pioneros en materializar todo un mundo de fantasía producido por los personajes de sus películas de cine y animación: en 1955 inauguró Disneyland iniciando así el camino a la construcción de parques temáticos basados en mundos construidos con las experiencias en la sala cinematográfica y en el hogar, gracias a la televisión.

Hoy día la iniciativa de Disney es imitada por las actividades de ocio digital que pretenden dar vida material a unos mundos nacidos, construidos y desarrollados en Internet, como es el proyecto de parque temático que la empresa propietaria de la marca Playfish tiene previsto inaugurar en el año 2014. El parque estará basado en las experiencias de ocio producidas durante el uso de aplicaciones de la marca Playfish en Facebook (ver playfish.wordpress. com, publicado el 1 de abril de 2011). Playfish es una de las marcas de mayor uso y prestigio de juegos sociales de Facebook después de Zynga cuyo juego social Farmville protagonizó el boom de los juegos sociales en el servicio de red social al obtener en el ejercicio del año 2009 unos ingresos de 250 millones de dólares americanos por venta de objetos digitales (Morrisen, 2010). El parque temático de Playfish, según anunciaban en una de sus páginas oficiales, se basará en sus tres juegos sociales más potentes y en un área temática que queda reservada a un juego social que todavía no ha sido lanzado al mercado digital (en el momento de escribir este artículo).

\section{La coproducción del ocio digital}

A los anteriores desafíos empresariales tenemos que añadir uno nuevo, ahora las empresas deben enfrentarse al reto de saber interpretar y adaptarse al creciente protagonismo de los individuos como coproductores de la actividad de ocio y de la misma experiencia de ocio. Red 2.0 es el nombre con el que algunos autores denominan a las tecnologías digitales que han incrementado la capacidad de interacción entre los individuos haciendo posible la coproducción del ocio digital (Holt, 2011; Regner et al., 2010 y Margaix, 2007).

La coproducción del ocio digital ha dado lugar a productos de ocio de éxito económico, prácticamente todos ellos basados en la interacción personificada en los mundos digitales: Facebook (y todos los juegos que en esa plataforma se desarrollan como Playfish, entre otros), Youtube, Flickr, etc., por nombrar a los más conocidos. Otras iniciativas fusionan el trabajo y el ocio, y no por ello han tenido menos favor por parte de los consumidores, como es el caso de la Wikipedia que no deja de ser la digitalización de una enciclopedia tradicional, pero esta vez el contenido es producido y rectificado por los mismos lectores; la fundación Wikipedia, no obstante, genera otros productos derivados que le proporcionan un rendimiento económico (aparte de las donaciones). Incluso otras iniciativas de coproducción llegan a integrar más los conceptos de trabajo y ocio, como son las relacionadas con los programas informáticos abiertos y libres ofrecidos bajo diversas licencias de Creative Commons.

La coproducción de las actividades y productos de ocio digital en la Red crea desafíos adicionales (Ritzer y Jurgenson, 2010). A diferencia de la producción tradicional, el resultado de la experiencia de ocio ya no depende sólo de la interacción con otros individuos, sino de la interacción entre éstos y con las mismas empresas responsables de las plataformas de ocio. La actividad de ocio evoluciona en constante interacción, como han puesto de manifiesto diferentes situaciones problemáticas entre Playfish y los mismos jugadores. La coproducción genera un valor compartido (un producto, una experiencia) que las empresas tratan de transformar en un valor económico. 
Las empresas ancladas en el modelo tradicional no han sabido responder al desafío digital. Los consumidores, no obstante, no han esperado y han iniciado su coproducción de obras derivadas: graban películas, las comparten, subtitulan y recomiendan, pero en plataformas privadas sin autorización de los productores originales. En otros casos, las empresas las toleran simplemente porque les facilita la segmentación de los mercados sin necesidad de incrementar las inversiones; este es el caso de la empresa india Notion Ink, productora de la tableta táctil Adam y la iniciativa notioninkhacks.com dedicada a liberar el sistema operativo y a ofrecer a los usuarios "lo último", lo que todavía no es oficial.

La coproducción en la que participan los individuos durante su tiempo de ocio es un recurso de doble filo. Dar voz al consumidor en la creación significa concederle cierto poder que a veces puede llegar a ser inmanejable, especialmente ahora con la capacidad de comunicación e interacción de la que disponen en la Red de Internet, como ya ha ocurrido según los datos proporcionados por Patterson (2011).

Otro reto es la gestión de unos derechos de propiedad en un mundo donde existe coproducción, tanto en iniciativas como Playfish, como en las obras derivadas (películas subtituladas, libros modificados, producciones musicales compartidas) (Grimes, 2006). Los derechos de propiedad y su ausencia traspasan las fronteras (Breen, 2010), y su alteración modifica la competitividad de las empresas y países (López y García, 2008). Los derechos de propiedad crean pequeños monopolios temporales con el objeto de estimular la innovación, pero su aplicación parece estar limitándola en beneficio de ciertas productoras. Iniciativas como la de Google Books para digitalizar las obras del fondo de las bibliotecas han topado con la resistencia de productores y editores que han conseguido alargar el monopolio temporal de obras cuyos derechos estaban a punto de expirar. En otras épocas de la historia la creación de obras derivadas era algo habitual que estimuló la creatividad (hoy logrado a través de las licencias Creative Commons). Obras derivadas pueden encontrarse en la red, reescrituras de grandes éxitos editoriales, por ejemplo, como la saga de Harry Potter.

Esto es, la coproducción supone un cambio de paradigma, pasamos de un mundo donde una organización produce y controla el contenido digital, el objeto, la actividad dirigida a unos individuos (Patterson, 2011), a otro en el que los individuos participan en la producción hasta el punto de convertir al consumidor en productor, consumidor y promotor del ocio digital (prosumer, lo llaman Ritzer y Jurgenson, 2010). Pese a que el sector audiovisual es el que tiene mayor peso en el mercado de los contenidos digitales en la era de Internet, el dominio de los modelos tradicionales de negocio basados en la doctrina de los derechos de autor dificultan su despegue según varios autores (Echeverría, 2009 y Breen, 2010). Probablemente estamos presenciando una época fértil en la creación procedente de los jóvenes artistas que ahora tienen una mayor autonomía en su producción y distribución, y, al mismo tiempo, una época de dificultades para las productoras tradicionales. Por lo tanto, su futuro dependerá de la manera en la que reaccionen a este desafío (FICOD, 2009, 2010).

\section{Conclusiones}

La evidencia que hemos presentado muestra cómo la introducción del ocio digital desafía tanto el concepto de ocio establecido como sus dimensiones, planteándose la necesidad de abordarlo desde una perspectiva holística que privilegia el estudio del individuo y su experiencia contextual, independientemente de que disfrute de sus actividades de ocio durante su tiempo libre en el mundo natural o en el digital. Las tradicionales propiedades dicotómicas del ocio se han alterado, al menos han dejado de ser dicotómicas cuando el tiempo de trabajo es penetrado por el de ocio, y al revés, debido a la "inmaterialidad" de las producciones digitales de ocio y de trabajo.

Diferencias en la exposición a las tecnologías digitales producen concepciones distintas de los mundos sociales naturales y de los mundos construidos digitalmente, cuyos extremos añaden una nueva dicotomía, mundos separados, mundos únicos. Según si los individuos se sitúan en los extremos 0 en posiciones intermedias, se observan transformaciones o tendencias en la experiencia del ocio: jóvenes con una concepción holística (nativos digitales), adultos viviendo en el mundo natural pero haciendo incursiones al construido digitalmente (inmigrantes digitales); individuos con posiciones sociales privilegiadas que tienen mayor exposición a las tecnología y por ello difieren no solo en su acceso sino también en el uso durante su tiempo de ocio;

ARBOR Vol. 188754 marzo-abril [2012] 395-407 ISSN: 0210-1963

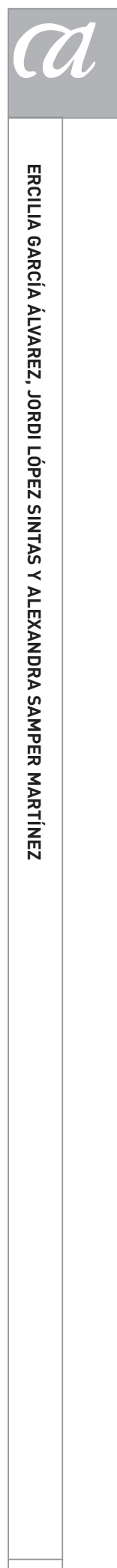

403 
individuos con una mayor interacción social presencial que utilizan las tecnologías digitales: para incrementar su ocio y su interacción, para construir una identidad social móvil, para desafiar y reproducir las construcciones sociales del ocio.

Finalmente, las empresas también difieren en su concepción de los mundos naturales y los construidos digitalmente. Las empresas tradicionales los conciben como mundos separados, por lo que sus propuestas empresariales se enfocan fundamentalmente en la digitalización de su oferta. Por contra, las empresas de origen digital y desde una concepción holística responden proactivamente a través de la doble propuesta de la materialización de la actividad digital y de la gestión de la coproducción de su oferta con los propios usuarios. Se da respuesta así a las nuevas tendencias introducidas por el ocio digital haciendo frente a sus propios retos empresariales.

\section{NOTA}

* Esta investigación se ha podido realizar gracias al soporte financiero de los proyectos de investigación EC0200804101 (Plan nacional l+D+i de del Ministerio de Ciencia e Innovación del Gobierno de España con el soporte del FEDER de la UE), 2009-SGR-411 (Generalitat de Catalunya) y del fondo de investigación del Centre d'Estudis i de Recerca d'Humanitats.

\section{REFERENCIAS}

Abbott-Chapman, J. y Robertson, M. (2002): "Youth, Leisure and Home: Space, Place and Identity", Loisir et Société Society and Leisure, 24(2), 485-506.

Bendon, M. (2011): "Coming in 2014: a Playfish amusement park!". Recuperado 2 de abril de 2011, desde http://playfish. wordpress.com/2011/04/01/comingin-2014-a-playfish-amusement-park/

Boczkowski, P. J. (2010): "Making sense of emerging phenomena and rethinking existing concepts", Information, Communication \& Society, 13 (4), 470484.

Bogart, L. (1989): Press and public: who reads what, when, where, and why in American newspapers, Hillsdale, N.J.: L. Erlbaum Associates.
Breen, M. (2010): "Digital determinism: culture industries in the USA-Australia Free Trade Agreement", New Media Society, 12, 657-676.

Bretonès, D. D.; Quinio, B. y Réveillon, G. (2010): "Bridging virtual and real worlds: enhancing outlying clustered value creations", Journal of Strategic Marketing, 18 (7), 613-625.

Bryce, J. (2001): "The technological transformation of leisure", Social Science Computer Review, 19(1), 7-16.

Bucy, E. P. (2000): "Social access to the Internet", The Harvard International Journal of Press/Politics, 5, 50-61.

Buse, C. E. (2009): "When you retire, does everything become leisure? Information and communication technology use and the work/leisure boundary in retirement", New Media \& Society, $11(7), 1143-1161$.

Calvert, S. L. (2005): "Cognitive effects of video games", en J. Raessens y J. Goldstein (eds.), Handbook of Computer Games Studies, Cambridge MA: MIT Press, pp. 125-131.

Campbell, S. W. y Kwak, N. (2010): "Mobile Communication and Civic Life: Linking Patterns of Use to Civic and Political Engagement", Journal of Communication, 60(3), 536-555.

Carr, D.; Schott, G.; Burn, A. y Buckingham, D. (2004): "Doing game studies: A multi-method approach to the study of textuality, interactivity, and narra-
Aceptado: 22 de julio de 2011 
tive space", Media International Australia Incorporating Culture and Policy, 110, 19-30.

Cassell, J. y Jenkins, H. (1998): "Chess for girls?: Feminism and computer games", en J. Cassel y H. Jenkins (eds.), From Barbie to Mortal Kombat: Gender and Computer Games, Cambridge, MA: MIT Press.

Colwell, J.; Grady, C. y Rhaiti, S. (1995): "Computer games, self-esteem, and gratification of needs in adolescents", Journal of Community and Applied Social Psychology, 5, 195-206.

Cox, A. M.; Clough, P. D. y Marlow, J. (2008): "Flickr: a first look at user behaviour in the context of photography as serious leisure", Information Research, 13 (1). Recuperado 11 abril de 2011, desde http://informationr.net/ir/13-1/ paper336.html.

Cruz, J. (2011): "El poder pasa del editor al lector", El País, 14 de marzo. (Recuperado el 14 de marzo de 2011, desde http://www.elpais.com/articulo/reportajes/poder/pasa/editor/lector/elpepus ocdmg/20110313elpdmgrep_4/Tes).

De Kort, Y. A. W.; IJsselsteijn, W. A. y Gajadhar, B. J. (2007): "People, Places, and Play: A research framework for digital game experience in a sociospatial context", en B. Akira (Ed.), Situated Play: Proceedings of the 2007 Digital Games Research Association Conference, Tokyo: The University of Tokyo, pp. 823-830. (Retrieved from http://www.digra.org/dl/display_ html?chid=07311.21038.pdf).

Delamere, F. M. y Shaw, S. M. (2008): "They see it as a guy's game: The politics of gender in digital games", Leisure/ Loisir, 32(2), 279.

DiMaggio, P. y Hargittai, E. (2001): "From the 'Digital Divide' to 'Digital Inequality': Studying Internet Use as Penetration Increases", Working Paper Series, 19, Princeton, N.J.: Center for Arts and
Cultural Policy Studies, Woodrow Wilson School, Princeton University.

DiMaggio, P.; Hargittai, E.; Celeste, C. y Shafer, S. (2004): "Digital inequality: From unequal access to differentiated use", en K. Neckerman (Ed.), Social inequality, New York: Russell Sage, pp. 355-400.

DiMaggio, P.; Hargittai, E.; Neuman, W. y Robinson, J. (2001): "Social implications of the Internet", Annual Review of Sociology, 27, 307-336.

Douglas, M. y Isherwood, B. (1996): The World of Goods, London, UK: Routledge.

Du, Y. y Martin, B. (2008): "Electronic Media and Leisure Time Reading: Responses of School Librarians", en IASL 2008 Conference. ISSN 0257-3229.

Echeverría, J. (2009): "Cultura digital y memoria en red", ARBOR Ciencia, Pensamiento y Cultura, CLXXXV 737 mayojunio, 559-567, ISSN: 0210-1963.

Engelen, J. J. (2008): "A Rapidly Growing Electronic Publishing Trend: Audiobooks for Leisure and Education", en L. Chan y S. Mornati (eds.), Open Scholarship: Authority, Community and Sustainability in the Age of Web 2.0. ELPUB2008 Conference on Electronic Publishing, Toronto, Canada.

English-Lueck, J. A. (1998): "Technology and social change: The effects on family and community", COSSA Congressional Seminar. Recuperado 19 de junio 2010 desde http://www.sjsu.edu/ depts/anthropology/svcp/CossaP.htm.

Fulton, C. (2009): "Quid Pro Quo: Information Sharing in Leisure Activities", Librarry Trends, 57(4), 753-768.

García Álvarez, E. y López-Sintas, J. (2008): "La cinematografía ante el reto audiovisual: Políticas para mejorar la eficiencia productiva y reducir los fallos de comercialización", en López, J. y Padrós, C. (Dirs.), Cinco ensayos de derecho y economía del cine, Barcelona: Editorial Atelier, pp. 11-42.
Gershuny, J. y Fisher, K. (1999): "Leisure", en Halsey, A. H. y Webb, J. (eds.), Twentieth Century British Social Trends (3 $3^{\text {rd }}$ Edition), London: MacmiIlan Publishers.

Griffiths, M. (1997): "Violent video games and aggression: A review of the literature", Aggression and Violent Behavior", 4, 203-212.

Griffiths, M. y Light, B. (2008): "Social networking and digital gaming media convergence: Classification and its consequences for appropriation", Information Systems Frontiers, 10, 447-459.

Grimes, S. M. (2006): "Online multiplayer games: a virtual space for intellectual property debates?", New Media \& Society, 8(6), 969-990.

Gunter, B. (2005): "Psychological effects of videogames", en J. Raessens y J. Goldstein (eds.), HandBook of Computer Games Games Studies, Cambridge, MA: MIT Press.

Hargittai, E. y Hinnant, A. (2008): "Digital Inequality: Differences in Young Adults' Use of the Internet", Communication Research, 35(5), 602-621.

Henwood, K. L. (1993): "Women and later life: The discursive construction of identities within family relationships", Journal of Aging Studies, 7(3), 303-319.

Holderness, M. (1994): Welcome to the global village, London: Royal Geographical Society.

Iso-Ahola, S. E. (1997): "A psychological analysis of leisure and health", en J. T. Haworth (Ed.), Work, leisure and well-being, New York: Routledge, pp. 117-130.

Jansz, J. y Martens, L. (2005): "Gaming at a LAN event: the social context of playing video games", New Media \& Society, 7(3), 333-355.

Johnson, N. F. (2009): "Cyber-relations in the Field of Home Computer Use for Leisure: Bourdieu and teenage tech- 
nological experts", E-Learning, 6(2), 187-197.

Johnson, T. J. y Kaye, B. K. (2003): "Around the World Wide Web in 80 Ways", Social Science Computer Review, 21(3), 304-325.

Jones, C.; Ramanau, R.; Cross, S. y Healing, G. (2010): "Net generation or Digital Natives: Is there a distinct new generation entering university?", Computers \& Education, 54, 722-732.

Juniu, S. (2009): "The transformation of leisure", Leisure/Loisir, 33(2), 463-478.

Jupp, B. y Bentley, T. (2001): "Surfing alone? E-commerce and social capital", en J. Wilsdon (Ed.), Digital Futures: Living in a Dot-com World, London: Earthscan, pp. 97-118.

Kelly, J. R. (1978): "A revised paradigm of leisure choices", Leisure Sciences: An Interdisciplinary Journal, 1(4), 345-363.

Kennedy, T. L. M. y Wellman, B. (2007): "The Networked Household", Information, Communication \& Society, 10(5), 645-670.

Kennedy, T. L. M.; Wellman, B. y Klement, K. (2003): "Gendering the digital divide", IT\&Society, 1(5), 72-96.

Khouja, M. y Wang, Y. (2010): "The impact of digital channel distribution on the experience goods industry". Recuperado 25 de mayo de 2011, desde http:// econpapers.repec.org/.

Kiesler, S.; Sproull, L. y Eccles, J. S. (1985): "Pool, halls, chips, and war games: Women in the culture of computing", Psychology of Women Quarterly, 9(4), 451-462.

Kozinets, R. V. (2010): Netnography, Doing Ethnographic Research Online, London: SAGE Publications Ltd.

Kraut, R.; Kiesler, S.; Boneva, B.; Cummings, J.; Helgeson, V. y Crawford, A. (2002): "Internet Paradox Revisited", Journal of Social Issues, 58(1), 49-74.

Kraut, R.; Patterson, M.; Lundmark, V.; Kiesler, S.; Mukopadhyay, T. y Scherlis, W.
(1998): "Internet paradox: A social technology that reduces social involvement and psychological well-being?", American Psychologist, 53, 1017-1031.

Leung, L. (2001): "College student motives for chatting on ICQ", New Media \& Society, 3(4), 483-500.

Leung, L. (2009): "User-generated content on the internet: an examination of gratifications, civic engagement and psychological empowerment", New Media \& Society, 11(8), 1327-1347.

Livingstone, S. M. (2002): Young People and New Media, London: Sage.

López-Sintas, J. (2011): "Acceso a las expresiones culturales e incentivos a la creación: Argumentos, evidencia e implicaciones", en Padros Reig, C., y López Sintas, J. (Dirs.), El canon digital a debate: Revolución tecnológica y consumo cultural en un nuevo marco jurídico-económico, Barcelona: Atelier, pp. 43-65.

López-Sintas, J.; Filimon, N. y Garcia-Álvarez, M. E. (2011): "A social theory of internet uses based on consumption sale and linkage needs", Social Science Computer Review, en prensa.

Mäntymäki, M. y Merikivi, J. (2010): "Investigating the Drivers of the Continuous Use of Social Virtual Worlds", 43rd Hawaii International Conference on System Sciences, Hawaii: IEEE Computer Society, pp. 1-10.

Margaix A. D. (2007): "Conceptos de web 2.0 y biblioteca 2.0: origen, definiciones y retos para las bibliotecas actuales", El profesional de la información, 16(2), 95-106.

Martinson, A. M.; Schwartz, N. y Vaughan, M. W. (2002): "Women's experiences of leisure", New Media \& Society, 4(1), 29-49.

Mennecke, B. E.; Triplett, J. L.; Hassall, L. M.; Conde, Z. J. y Heer, R. (2011): "An Examination of a Theory of Embodied Social Presence in Virtual Worlds", Decision Sciences, 42(2), 413-450.
Mokhtarian, P. L.; Salomon, I. y Handy, S. L. (2006): "The Impacts of Ict on leisure Activities and Travel: A Conceptual Exploration", Transportation, 33(3), 263-289.

Morrissey, B. (2010): "Game on for Brands: Marketers get serious about social gaming", Adweek, 51. Recuperado 15 de enero de 2010, desde http://www. adweek.com/news/technology/gamebrands-101281.

Neulinger, J. (1974): The psychology of leisure, Springfield, IL: Charles C Thomas.

Orchard, L. J. y Fullwood, C. (2010): "Current Perspectives on Personality and Internet Use", Social Science Computer Review, 28(2), 155-169.

Patterson, A. (2011): "Social-networkers of the world, unite and take over: A meta-introspective perspective on the Facebook brand", Journal of Business Research, en prensa.

Pereira, J. (1994): "Computers: The Gender Divide: A Tool for Women, a Toy for Men: Video Games Helps Boys Get a Head Start", Wall Street Journal, 15 March: B1.

Prensky, M. (2001a): "Digital Natives, Digital Immigrants". Recuperado 1 de febrero de 2011 de On the Horizon (MCB University Press, Vol. 9, No. 5), URL: www.marcprensky.com.

Prensky, M. (2001b): "Digital Natives, Digital Immigrants, Part II: Do They Really Think Differently?". Recuperado 1 de febrero de 2011 de On the Horizon (MCB University Press, Vol. 9, No. 6), URL: www.marcprensky.com.

Putnam, R. D. (2000): Bowling Alone. The collapse and revival of American community, New York: Simon and Schuster.

Regner, T.; Barria, J. A.; Pitt, J. V. y NeviIle, B. (2010): "Governance of digital content in the era of mass participation", Electronic Commerce Research, 10, 99-110. 
Ritzer, G. y Jurgenson, N. (2010): "Production, Consumption, Prosumption", Journal of Consumer Culture, 10(1), 13-36.

Roberts, D. F.; Foehr, U. G. y Rideout, V. J. (2005): Generation M: Media in the Lives of 8-18 year-olds, Menlo Park, CA: Kaiser Family Foundation.

Robinson, J. P. y Godbey, G. (1997): Time for Life, University Park: Pennsylvania State University Press.

Rosedale, P. (2007): "Prefacio", Second Life the official guide, New Jersey: Wiley \& Sons, Inc.

Schroeder, R. (2010): "Mobile phones and the inexorable advance of multimodal connectedness", New Media \& Society, 12(1), 75-90.
Selwyn, N. (2004): "Reconsidering Political and Popular Understandings of the Digital Divide", New Media Society, 6(3), 341-362.

Siddiqui, Shakeel y Darach Turley (2006): "Extending the Self in a Virtual World", Advances in Consumer Research, 33, 647-648.

Stald, G. (2011): "Mobile Identity: Youth, Identity, and Mobile Communication Media", en David Buckingham (ed.), Youth, Identity, and Digital Media. The John D. and Catherine T. MacArthur Foundation Series on Digital Media and Learning, Cambridge, MA: The MIT Press, 2008, 143-164. doi 10.1162/ dmal.9780262524834.143.
Stebbins, R. A. (1992): Amateurs, Professionals, and Serious Leisure, Montreal: McGill-Queen's University press.

Stebbins, R. A. (1997): "Casual leisure: a conceptual statement", Leisure Studies, 16(1), 17-25.

Turgeman-Goldschmidt, 0. (2005): "Hackers' Accounts", Social Science Computer Review, 23(1), 8 -23.

Wilson, J. (1980): "Sociology of Leisure", Annual Review of Sociology, 6, 21-40.

Zillien, N. y Jäckel, M. (2007): "Digital Inequalities-Not just a Passing Phenomenon", Anual Meeting of the International Communication Association, TBA, San Francisco. 